\title{
LOS CULTIVADORES DE LA CIENCIA ESPANNOLES Y EL GOBIERNO DE JOSÉ I (1808- 1813). UN ESTUDIO PROSOPOGRÁFICO
}

\author{
José Ramón Bertomeu Sánchez
}

El estudio de las actitudes políticas de los cultivadores de la ciencia españoles durante el siglo XIX constituye un tema de gran interés para conocer las condiciones en las que se desarrolló la actividad científica en la sociedad española de este período. Una de las razones que habitualmente suele proponerse para explicar el "período de catástrofe» de la ciencia española del primer tercio del siglo XIX es la represión sobre afrancesados y liberales por parte del gobierno de Fernando VII. Como ejemplos de esta represión se suele señalar los problemas que sufrieron algunos autores científicos españoles con una importante producción impresa, tales como Juan Manuel de Aréjula o Antonio de Gimbernat, cuyas biografías son bastante conocidas. Sin embargo, cuando se trata de profundizar en este tema, ampliando el número de biografías consideradas, rápidamente se comprende que nuestros conocimientos son todavía muy limitados debido, en parte, a varias dificultades propias de este tipo de investigación. En primer lugar, es necesario reconocer el escaso conocimiento que tenemos sobre muchos de los cultivadores de la ciencia españoles del siglo XIX, especialmente aquéllos cuya producción impresa es poco importante o aquéllos que no ocuparon cargos dentro de las instituciones científicas. Además, muchos de los datos que conocemos proceden de trabajos con un marcado carácter hagiográfico, tales como discursos pronunciados en instituciones académicas o notas necrológicas en 
revistas científicas. Como se puede comprobar fácilmente, en este tipo de escritos se suele evitar la narración de algunas características del biografiado que son consideradas como "deshonrosas» por el autor del escrito, por ejemplo, la colaboración con el gobierno de José I. Finalmente, también cabe señalar una última dificultad, la inherente al estudio de las actitudes políticas de un conjunto heterogéneo de individuos, los cultivadores de la ciencia, de los que suele estudiarse, con mayor detalle, sus aportaciones científicas. Como es lógico, en la mayor parte de los casos resulta muy difícil conocer la actitud política de estos individuos a través de las fuentes habitualmente más utilizadas por los historiadores de la ciencia como las publicaciones científicas, los archivos de instituciones científicas, etc.

En este trabajo se ofrece el resultado de un estudio prosopográfico basado en un conjunto de 483 biografías de cultivadores de la ciencia españoles que vivieron durante el período de la Guerra de la Independencia (1808-1814). El objetivo perseguido es efectuar una aproximación a la actitud política de este grupo de individuos respecto al gobierno de José I, dentro de una investigación más amplia sobre la actividad científica española de este período (1). En primer lugar, expondremos los materiales y métodos empleados para, a continuación, presentar los principales resultados de este trabajo.

\section{Material y métodos}

El uso de la prosopografía en los estudios históricos sobre la ciencia ha permitido contestar adecuadamente muchas cuestiones planteadas por la investigación histórico-social (2). Se trata de una técnica utilizada en numerosas investigaciones sobre la historia de la medicina y la historia de la ciencia española dentro del Instituto de Estudios Documentales e Históricos sobre la Ciencia de Valencia (3). De modo breve, describiremos las características particulares de nuestro análisis prosopográfico comenzando por los criterios utilizados para confeccionar la nómina de "cultivadores de la ciencia» sobre la que hemos basádo nuestro estudio.

Los estudios sobre las profesiones y ocupaciones científicas muestran que durante el período que estamos analizando se produjo un importante cambio en algunos países europeos en el rol del científico dentro de la sociedad, especialmente tras los procesos revolucionarios de Francia. Al margen de las discusiones sobre el nivel de institucionalización de la acti- 
vidad científica en la España de la época, resulta necesario tener en cuenta que la actividad científica no constituía una profesión, al menos en el sentido que se le ha dado habitualmente a este término en los estudios histórico-sociales sobre la ciencia (4). En general, la situación varía según el área científica considerada, siendo bastante diferente en áreas como la medicina y la farmacia, cuya actividad se encontraba mucho más regulada, frente a otras como la química o la agronomía, mucho menos institucionalizadas. Por todo ello, al hablar de actividad científica nos estamos refiriendo a un grupo heterogéneo de tareas realizadas por individuos con distintas profesiones y ocupaciones, a los que no parece adecuado agrupar bajo términos como "científicos» o "comunidad científica».

En estas circunstancias, ha sido necesario establecer un criterio'general para incluir o excluir a un individuo de la época dentro del grupo que hemos considerado como "cultivadores de la ciencia». Este criterio ha sido la publicación de al menos un impreso referente a cualquiera de las ciencias de la naturaleza, incluyendo la medicina, cirugía y farmacia (5). De acuerdo con Solla Price, consideramos que el acto de la publicación constituye un producto esencial de la actividad científica contemporánea, mediante el cual una idea puede ser evaluada, aceptada o corregida por el resto de los cultivadores de la ciencia en un período determinado (6). No obstante, hemos incluido en nuestra lista de cultivadores de la ciencia un reducido número de individuos de los que no conocemos publicación alguna, pero que hemos considerado necesaria su inclusión de acuerdo con otros criterios, tales como su importante producción manuscrita, sus cargos institucionales, o sus aportaciones técnicas. En cualquier caso, este último grupo representa una porción muy reducida del total por lo que nos referiremos indistintamente al conjunto como "cultivadores de la ciencia» o «autores científicos».

Aclarado este primer aspecto, debemos señalar los límites temporales y geográficos dentro de los cuales hemos realizado nuestra selección de autores científicos. Hemos considerado todos aquellos autores que, nacidos antes de la última década del siglo XVIII, continuaban vivos durante los años de la Guerra de la Independencia (1808-1814) y aparecen citados en alguno de los repertorios bibliográficos y biobibliográficos utilizados. En aquellos casos en los que sólo disponemos de una referencia aislada hemos incluido a aquéllos en que esta referencia se situaba dentro de un intervalo de 10 años alrededor de la guerra. Entendemos como «autor español» a todos aquellos autores que, o bien nacieron en los territorios de 
la corona española de ese momento, o bien se formaron científicamente y desarrollaron la mayor parte de su actividad científica en España.

Los repertorios bibliográficos y biográficos utilizados han sido los que indicamos en la bibliografía final. En general, podemos clasificar estos trabajos en dos grupos:

1. Repertorios bibliográficos y biobibliográficos de autores científicos españoles: estos repertorios los hemos revisado completos dentro de nuestros límites temporales. Además del Diccionario histórico sobre la ciencia moderna en España publicado por López Piñero, Glick, Navarro y Portela (1983) hemos consultado diccionarios biobibliográficos dedicados a áreas científicas determinadas como el de Roldán Guerrero (1958-1976) para la farmacia, Hernández Morejón (1842-52), Chinchilla (1841-46), Alvarez Sierra (1963), Calbet-Corbella (1981-83) para la medicina y cirugía, Colmeiro (1858) para la botánica, Palau Claveras (1973) para la veterinaria, Maffei y Rua (1871-72) para la mineralogía, Fernández de Navarrete (1851) para la náutica, Antón Ramírez (1865) para la agronomía, Bonet Correa (1980) para la ingeniería civil y Capel et al. (1983) para los ingenieros militares. También hemos consultado dos importantes archivos personales que se encuentran en el museo historicomédico del Instituto de Estudios Documentales e Históricos sobre la Ciencia: el archivo de Rodrigo de Pertegás (7) que contiene abundante información sobre los médicos valencianos y el inédito diccionario biográfico médico y quirúrgico de Sánchez Quintanar (8).

2. Repertorios biobibliográficos regionales y diccionarios biográficos generales de historia de España: con las listas de autores científicos elaboradas en el apartado anterior hemos efectuado entradas en los repertorios biobibliográficos regionales como el de Torres Amat (1836), Corminas (1849) y Elías de Molins (1972) para los escritores catalanes, Pastor Fuster (1827-1830) para Valencia, Bover (1868) para Baleares, Latassa (1884-1886) para Aragón y Suárez para Asturias (1939-1959). También nos ha sido de gran utilidad el Diccionario Histórico del Trienio Liberal dirigido por Gil Novales (1991) que, por su proximidad temporal, recoge mucha información sobre las biografías de nuestro interés. Finalmente, hemos consultado también diccionarios históricos generales como el de Artola (1991) o las biografías que aparecen en la enciclopedia universal ilustrada (ESPASA). 
Hemos completado la información existente con estudios biográficos particulares sobre cultivadores de la ciencia y trabajos dedicados a instituciones científicas españolas de principios del siglo XIX (9).

Junto con todo este material bibliográfico, hemos analizado las referencias a los cultivadores de la ciencia que aparecen entre la documentación del gobierno afrancesado que hemos estudiado (10). Además de la producción legislativa impresa (11), hemos consultado los números de la Gaceta de Madrid que, durante estos años, se publicó diariamente. También hemos consultado diversos documentos que se encuentran en varios archivos españoles y franceses que corresponden, principalmente, a varios expedientes tramitados por el Ministerio del Interior y el Consejo de Estado de José I los cuales contienen numerosos datos sobre la actividad científica española de esos años (12). Estos documentos se encuentran principalmente en el Archivo del Palacio Real (13), Archivo Histórico Nacional (14), Archivo General de Simancas (15), Archivo de la Universidad de Madrid (16) y Archivos Nacionales de Francia (17). También hemos analizado varios libros de registros de expedientes del Ministerio del Interior que contienen numerosas referencias a expedientes perdidos o no localizados referentes a la gestión de varias instituciones científicas (18). Finalmente, no podemos acabar esta larga enumeración de fuentes sin citar la conocida nómina de intelectuales afrancesados que reprodujo Danvila y Collado en su obra Del poder civil en España (19).

Toda esta información ha sido analizada con el objetivo obtener un perfil biográfico colectivo de los cultivadores de la ciencia españoles de este período. Hemos centrado nuestra atención sobre su lugar de residencia durante la guerra, su formación científica, las materias científicas cultivadas y su ocupación o profesión. Finalmente, en el último apartado, analizamos la actitud de estos autores científicos frente al gobierno afráncesado.

2. Análisis prosopográfico de los cultivadores de la ciencia españoles (18081813)

\subsection{Edad de los cultivadores de la ciencia}

Con el objetivo de analizar las características de las 483 biografías de cultivadores de la ciencia que hemos estudiado, vamos a establecer en primer lugar una distribución por grupos, de acuerdo con la edad que tenían 
durante la Guerra de la Independencia (1808-1814) (20). Un primer grupo está formado por los supervivientes del período de la Ilustración, todos ellos con más de 40 años durante la guerra, que habían realizado la mayor parte de su producción científica antes de 1808 y, en muchos casos, ocupaban diversos puestos en instituciones científicas y docentes. Este grupo constituye alrededor de un tercio $(30,4 \%)$ de nuestro conjunto de biografías. Otro grupo de aproximadamente un tercio $(30,0 \%)$ está formado por los nacidos entre 1770 y 1785 , que contaban entre 25 y 40 años durante la guerra. Una parte importante de ellos se había formado en las nuevas instituciones científicas de la Ilustración, dentro de las cuales se habían integrado en algunos casos. Muchos de los cultivadores de la ciencia de este grupo habían publicado algunas obras antes de 1808 y continuaron publicando durante y después de la guerra. Por último, el grupo de los más jóvenes está constituido por aquéllos que nacieron después de 1785, los cuàles aún no habían completado su formación al comienzo de la guerra y no comenzaron su producción científica hasta después de 1808. Dentro de nuestra nómina constituye un grupo mucho más reducido a los anteriores $(13,3 \%)$, debido, principalmente, a las condiciones que hemos establecido, esto es, al menos una obra impresa en los años de la guerra o en los años inmediatamente anteriores o posteriores.

Finalmente, nos queda un grupo de 127 cultivadores de la ciencia $(26,31 \%)$ de los que no tenemos suficientes datos para incluirlos en uno de los tres anteriores grupos. De ellos, 25 pertenecen al grupo $1 .^{\circ}$ ó $2 .^{\circ}(5,2 \%)$ y $33(6,9 \%)$ al grupo $2 .^{\circ}$ ó $3 .^{\circ}$ quedando un resto de $69(14,29 \%)$ imposibles de clasificar, ya que no disponemos de datos suficientes sobre sus biografías.

\subsection{Residencia de los cultivadores de la ciencia}

En la tabla I hemos tratado de establecer el lugar de residencia de los cultivadores de la ciencia españoles durante el período de 1808 a 1814. Dejando de lado los 89 casos en los que desconocemos el lugar de residencia durante esta época, hemos obtenido un total de 407 referencias a 106 ciudades y pueblos en los que residieron estos autores científicos a lo largo de estos años.

Un grupo reducido a cuatro ciudades completa casi la mitad (47\%) del total de residencias contabilizadas. En el lado opuesto, un grupo de casi tres cuartas partes del total de las ciudades y pueblos sólo suman un 19\% 
TABLA 1

Lugar de residencia de los cultivadores de la ciencia

\begin{tabular}{lcc}
\hline Población & $\begin{array}{c}\text { N. }{ }^{\circ} \text { de } \\
\text { residencias }\end{array}$ & \% acumulado \\
\hline Madrid & 100 & \\
Cádiz & 43 & 24,51 \\
Valencia & 26 & 35,05 \\
Barcelona & 25 & 41,42 \\
Sevilla & 13 & 47,55 \\
México & 12 & 50,74 \\
Palma de Mallorca & 10 & 53,68 \\
La Habana & 9 & 56,13 \\
Zaragoza & 9 & 58,33 \\
Lima & 8 & 60,54 \\
Santiago de Compostela & 7 & 62,50 \\
Almadén & 5 & 64,22 \\
Córdoba & 5 & 65,44 \\
Murcia & 5 & 66,67 \\
Oviedo & 5 & 67,89 \\
Tarragona & 5 & 69,12 \\
Valladolid & 5 & 70,34 \\
Vich & 5 & 71,57 \\
Bogotá & 4 & 73,79 \\
Mahón & 4 & 73,77 \\
Cinco poblaciones & 15 & 74,75 \\
Siete poblaciones & 14 & 78,43 \\
Setenta y cuatro poblaciones & 74 & 81,89 \\
Sian & & 100,00 \\
\hline
\end{tabular}

Fuente: BERTOMEU SANCHEZ (1993).

del total de referencias. Por lo tanto, podemos afirmar que los cultivadores de la ciencia durante este período se concentraron alrededor de un número reducido de ciudades, destacando entre ellas Madrid, Cádiz, Valencia y Barcelona.

La ciudad más importante en cuanto a número de residencias es Madrid que reúne la cuarta parte de las mismas, seguida por Cádiz con un número mucho menor (10\%). Se trata de las dos ciudades donde residie- 
ron los dos gobiernos civiles de la España de este período, esto es, el gobierno de José I, en Madrid, y el de la Regencia del Reino, en Cádiz. Por su parte, Valencia fue una ciudad que permaneció durante bastante tiempo fuera del dominio francés hasta que, en enero de 1812, el mariscal Suchet tomó la ciudad manteniendo allí su poder hasta 1813. Además, a finales de 1812, el gobierno de José I y su séquito se trasladaron a Valencia huyendo de las tropas inglesas y españolas que habían conquistado Madrid. Un caso muy diferente es el de Barcelona, que se mantuvo bajo un mando militar francés durante toda la guerra, llegando a formar parte del imperio napoleónico en los últimos años de la contienda bélica (21).

Del resto de ciudades, merece destacarse Palma de Mallorca que sirvió de refugio a muchos de los que huían de las tropas francesas, entre ellos varios cultivadores de la ciencia como Francisco Carbonell y Bravo, Pedro Castelló o José Mariano Vallejo. También destaca el caso del pueblo catalán de Vich, donde se reunieron diversos médicos y cirujanos militares, por ser una sede del Cuartel General del Ejército Español que luchaba contra las tropas francesas.

El número de residencias que corresponde a las colonias españolas es de $45(11 \%)$. Por lo general, se trata de cultivadores de la ciencia que vivieron la mayor parte de su vida en estos territorios y, por ello, la situación política del momento les afectó de un modo muy diferente que a los residentes en la metrópolis. En menor medida, también se puede encontrar entre estos autores científicos algunos que se hallaban provisionalmente en estas colonias, realizando una misión científica o militar, y que, posteriormente, regresaron a la península.

Las referencias a residencias en otros países son muy escasas sumando en total $22(5 \%)$. El país más importante es Francia con siete residencias, una parte de los cuales son algunos pensionados españoles en París como José María San Cristóbal, Mateo Orfila o José Radón. En la península italiana (seis residencias) encontramos a los supervivientes de los jesuitas expulsados durante el reinado de Carlos III que se habían establecido en diferentes ciudades de la península itálica, especialmente en Roma. Por el contrario, en Inglaterra (cinco residencias) residieron algunos autores científicos comisionados por el gobierno de Cádiz, sobre todo con misiones relacionadas con la astronomía y la náutica, aprovechando las buenas relaciones con el gobierno aliado inglés. El resto de los países tienen escaso peso en esta estadística y su aparición en esta tabla responde, más bien, a biografías muy particulares como la de Ferrer y Cafranga (EE.UU.) o Agustín de Betancourt (Rusia). 
Finalmente, nos interesa destacar una característica común a muchos de los cultivadores de la ciencia que estamos estudiando: el desplazamiento de su lugar de residencia habitual. Al menos 97 de los estudiados residieron en varias ciudades y pueblos durante la guerra, trasladándose de un lugar a otro según determinadas circunstancias. En muchos casos, se trataba de miembros del ejército que se vieron obligados a desplazarse de acuerdo con las exigencias militares de cada momento. En otros casos, algunos de estos autores científicos abandonaron su residencia debido a la ocupación de las tropas francesas o para evitar prestar juramento a José I, que fue exigido a todos los empleados públicos, entre ellos algunos miembros de las instituciones científicas de Madrid (22). A todo este grupo, hay que añadir los partidarios de José I que tuvieron que seguir al ejército francés en retirada y, en algunos casos, se vieron obligados a exilarse en Francia durante varios años.

\subsection{Formación científica de los cultivadores de la ciencia'}

Debido al deficiente conocimiento disponible sobre las biografías de muchos de los cultivadores de la ciencia de nuestro período, no disponemos de suficiente información sobre los establecimientos educativos en los que se formaron. A pesar de ello, ofreceremos los resultados que hemos obtenido esperando que nuevas investigaciones puedan ampliar el conocimiento de este aspecto. Del total de los cultivadores de la ciencia de nuestra nómina, existen 244 sobre los que no tenemos datos respecto al establecimiento en el que recibieron su formación científica. Los restantes 239 estudiaron en los establecimientos que hemos señalado en la tabla II, la cual debe interpretarse teniendo en cuenta que un mismo individuo puede haber sido contabilizado en varias instituciones docentes donde se formó.

El análisis de la tabla II muestra que la mayor parte de los cultivadores de la ciencia de los que tenemos información tenían una formación universitaria que habían adquirido en un total de 30 universidades diferentes, las cuales aparecen detalladas en la tabla III. Destacan tres universidades de la Corona de Aragón (Valencia, Cervera y Huesca) que abarcan casi la mitad $(47 \%)$ de las referencias a estudios universitarios. Aunque la mayor parte de las referencias corresponden a universidades españolas, destaca el gran número de estudios en universidades francesas (17) que suponen la décima parte del total de estudios universitarios $(11,87 \%)$. Esta situa- 
TABLA II

Instituciones en las que se formaron los cultivadores de la ciencia españoles

\begin{tabular}{lrr}
\hline Institución & $\begin{array}{c}N .^{\circ} \text { de } \\
\text { autores }\end{array}$ & $\%$ \\
\hline Universidades & 156 & 48,15 \\
Colegios de Cirugía & 40 & 12,35 \\
Academias Militares & 28 & 864 \\
Jardín Botánico de Madrid & 13 & 4,01 \\
Real Estudio de Mineralogía & 9 & 2,78 \\
Laboratorios de química de Madrid & 9 & 2,78 \\
Colegios de Farmacia & 8 & 2,47 \\
Escuela de Medicina Práctica de Madrid & 7 & 2,16 \\
Academia de Minas de Almadén & 5 & 1,54 \\
Junta de Comercio de Barcelona & 5 & 1,54 \\
Escuela de Ingenieros de Caminos & 3 & 0,93 \\
Escuela de Veterinaria de Madrid & 3 & 0,93 \\
Escuela de Veterinaria de Alfort & 2 & 0,62 \\
Observatorio Astronómico & 1 & 0,31 \\
Otros $\quad 35$ & 10,80 \\
\hline TOTAL & 324 & 100,00 \\
\hline
\end{tabular}

Fuente: BERTOMEU SANCHEZ (1993).

ción viene motivada por el importante papel de la Universidad de Montpellier, donde estudiaron varios cultivadores de la ciencia españoles, especialmente médicos. Tanto las universidades de las colonias españolas como las de otros países europeos (Inglaterra e Italia), tuvieron poca importancia en la formación de los cultivadores de la ciencia españoles.

El siguiente grupo en cuanto a importancia en la educación de los cultivadores de la ciencia españoles son los Colegios de Cirugía creados durante el siglo XVIII. Las 38 referencias se reparten, a partes casi iguales, entre los Colegios de Barcelona (17), Cádiz (11) y Madrid (11), mientras que en el de Mallorca sólo estudió uno de los autores científicos de nues- 
TABLA III

Universidades en las que se formaron los cultivadores de la ciencia españoles

\begin{tabular}{lcc}
\hline Universidades & $\begin{array}{c}N .^{\circ} \text { de } \\
\text { autores }\end{array}$ & \% acumulado \\
\hline Valencia & 32 & 20,51 \\
Cervera & 30 & 39,74 \\
Huesca & 12 & 47,44 \\
Alcalá & 12 & 55,13 \\
Montpellier & 10 & 61,54 \\
Salamanca & 7 & 66,03 \\
Zaragoza & 7 & 70,51 \\
Mallorca & 6 & 74,36 \\
Santiago & 6 & 78,21 \\
Oviedo & 5 & 81,41 \\
Sevilla & 3 & 83,33 \\
Paris & 3 & 85,26 \\
Avila & 2 & 86,54 \\
Gandia & 2 & 87,82 \\
La Habana & 2 & 89,10 \\
San Marcos de Lima & 2 & 90,38 \\
Toledo & 2 & 91,67 \\
$\quad$ Otras universidades & 13 & 100,00 \\
\hline TOTAL & & \\
\hline
\end{tabular}

Fuente: Bertomeu SÁNCHEz (1993).

tra lista. Mucha menor importancia tienen los Colegios de Farmacia de Madrid (6) y Barcelona (2) que fueron creados con posterioridad a los de Cirugía. También hemos recogido referencias a las Escuelas de Medicina Práctica establecidas en Madrid (7) y Barcelona (1) donde, durante algún tiempo, fue obligatorio realizar dos años de práctica antes de poder ejercer la medicina.

Otro grupo importante lo constituyen las Academias Militares con un total de 28 referencias. Las principales Escuelas Militares, sin contar los Colegios de Cirugía de la armada, son las de Guardamarinas de Cádiz (6), 
Cartagena (3) y el Ferrol (2), así como la de Artillería de Segovia (5), entre otras. En estas instituciones, se formaron principalmente militares que realizaron aportaciones a las matemáticas, la náutica y la astronomía, como principales áreas.

El último grupo lo constituyen un conjunto de diferentes instituciones creadas en su mayor parte en las décadas finales del siglo XVIII y que estaban destinadas, entre otras cosas, a ofrecer docencia en diversas áreas científicas como la botánica, la química, la mineralogía, la veterinaria o la ingeniería civil. Destaca también la labor desarrollada por la Junta de Comercio de Barcelona con sus cátedras de náutica y de química.

Otro aspecto que resulta muy interesante analizar son los estudios realizados en el extranjero por los cultivadores de la ciencia españoles. Como es sabido, a lo largo del siglo XVIII el gobierno español y otras instituciones subvencionaron el viaje de varios jóvenes cultivadores de la ciencia para que completaran su formación científica en el extranjero, en muchas ocasiones al lado de importantes autores científicos. En otros casos, un autor de obras científicas ya consagrado fue enviado a diversos países con el objetivo de realizar alguna misión científica determinada. Lamentablemente, no disponemos de un estudio global sobre las características de estos viajes científicos que han sido considerados como una de las bases de la política científica ilustrada. Veamos, a continuación, los datos de que disponemos obtenidos de las biografías que estamos estudiando.

$\mathrm{Al}$ menos 74 cultivadores de la ciencia de nuestra lista realizaron viajes científicos al extranjero, bien como pensionados, bien con alguna misión científica concreta. La mayor parte de los viajes se realizaron a Francia $(61,05 \%)$ y, en menor medida, a Inglaterra $(22,11 \%)$. Aunque los datos de que disponemos no nos permiten obtener conclusiones precisas, es interesante señalar que existen algunas diferencias entre las materias científicas cultivadas por los pensionados en Francia y los pensionados en Inglaterra. La mayor parte de los que viajaron a Francia eran cultivadores de la medicina y la química, aunque también podemos encontrar a cultivadores de otras áreas como la historia natural o las matemáticas, principalmente. Por el contrario, entre los pensionados en Inglaterra destaca el grupo de los cultivadores de la historia natural, seguidos por los cultivadores de la medicina y la química. En un nivel de importancia mucho menor se colocan los actuales territorios centroeuropeos de Alemania $(8,42 \%)$ y Hungría $(3,16 \%)$ que fueron los principales lugares de destino para los estudiosos de la mineralogía y minería, principalmente en la Escuela de Minas 
de Freiberg. El resto de los países, con la excepción de Italia (3,16\%), carecen de importancia real como receptores de este tipo de viajes científicos.

\subsection{Areas científicas cultivadas}

Utilizando la clasificación por materias científicas que hemos comentado en el apartado de "material y métodos», hemos estudiado las áreas científicas cultivadas prioritariamente por los autores científicos que estamos estudiando. Para realizar este estudio, nos hemos basado en los datos aportados por los repertorios biobibliográficos utilizados y, en su defecto, por el título de los libros publicados por nuestros autores. Los resultados

TABLA IV

Materias cultivadas por los autores científicos

\begin{tabular}{lrr}
\hline Materias científicas cultivadas & N. ${ }^{\circ}$ autores & $\%$ \\
\hline & 247 & \\
Medicina, cirugía y farmacia & 71 & 11,51 \\
Química y sus aplicaciones & 52 & 8,74 \\
Historia natural & 40 & 6,72 \\
Matemáticas & 36 & 6,05 \\
Geografía y cartografía & 28 & 4,71 \\
Astronomía & 27 & 4,54 \\
Agronomía y zootecnia & 24 & 4,03 \\
Náutica & 19 & 3,19 \\
Ingeniería civil & 15 & 2,52 \\
Veterinaria & 14 & 2,35 \\
Física & 9 & 1,51 \\
Ingeniería militar & 7 & 1,18 \\
Historia de la ciencia & 5 & 0,84 \\
Ciencia en general & 1 & 0,17 \\
Ciencias extraacadémicas & & \\
\hline
\end{tabular}

Fuente: Bertomeu SANCHEz (1993).

Asclepio-Vol. XLVI-1-1994 
están reflejados en la tabla IV, que debe ser analizada teniendo en cuenta que un autor científico pudo cultivar más de un área científica.

Estos resultados son muy semejante a la distribución por materias de las publicaciones científicas españolas durante la Guerra de la Independencia que hemos estudiado en otro trabajo (23). Como es habitual en este tipo de estudios, la medicina fue la materia científica cultivada por un mayor número de individuos de nuestra lista, puesto que un 41,51\% de los mismos realizaron alguna producción científica médica. También destaca el importante número de cultivadores de la química $(11,93 \%)$ y la historia natural $(8,74 \%)$, bastante superior a los porcentajes de estas materias que aparecen en nuestro estudio sobre la producción científica española entre 1808 y 1814 (24).

\subsection{Profesiones y ocupaciones de los cultivadores de la ciencia}

Como hemos comentado anteriormente, nuestra hipótesis de partida ha sido considerar que los cultivadores de la ciencia españoles de principios del XIX desarrollaron su producción científica desde diversas profesiones y ocupaciones, no existiendo la profesión de "científico», tal y como la entendemos en la actualidad. En la tabla $\mathrm{V}$ hemos tratado de resumir la información disponible sobre las profesiones y ocupaciones de los cultivadores de la ciencia estudiados.

Como se puede comprobar, una buena parte de ellos estaban dedicados a las diversas ocupaciones y profesiones sanitarias, destacando aquéllos que ejercían la profesión médica que son los más numerosos. Hemos diferenciado un reducido subconjunto bajo el epígrafe "Cargos administración sanitaria» para recoger a todos aquellos empleados en el Protomedicato y las diferentes Juntas Gubernativas de Medicina, Cirugía y Farmacia.

El siguiente grupo más numeroso lo constituyen aquellos cultivadores de la ciencia que impartían clases de diversas materias en los diferentes niveles de la educación, desde los profesores de primeras letras hasta los catedráticos de universidad y profesores de instituciones científicas. Merece señalarse el pequeño papel reservado a las Universidades (19 biografías), entre las que sólo destaca la Universidad de Valencia donde impartieron clases cinco de los individuos estudiados. Mucho más importante es el peso de diversas instituciones científicas creadas durante la Ilustración española y que impartían docencia en diversas materias como la ci- 
TABLA V

Ocupaciones y profesiones de los cultivadores de la ciencia

\begin{tabular}{lrr}
\hline Ocupaciones y profesiones & $N{ }^{\circ}$ & $\%$ \\
\hline Ocupaciones y profesiones sanitarias & 137 & 35,86 \\
$\quad$ Médicos (81) & & \\
$\quad$ Boticarios (20) & & \\
$\quad$ Cirujanos (20) & 112 & \\
$\quad$ Cargos administración sanitaria (16) & & 29,32 \\
Profesores & & \\
$\quad$ Universidad (19) & 56 & \\
$\quad$ Instituciones educación científica (70) & 29 & 14,66 \\
$\quad$ Otros establecimientos educativos (23) & 12 & 3,59 \\
Militares & 4 & 1,05 \\
Eclesiásticos & 3 & 0,79 \\
Juristas & 29 & 7,59 \\
Ingenieros civiles & & \\
Albéitares & & 100 \\
Otras ocupaciones y profesiones & 382 & \\
\hline TOTAL & & \\
\hline
\end{tabular}

Fuente: Bertomeu SANCHEZ (1993).

rugía, la botánica, la farmacia, la veterinaria, la mineralogía, etc. Entre todas ellas, destacan los Colegios de Cirugía (24 biografías), especialmente los de Cádiz (7), Madrid (7) y Barcelona (6). También encontramos cultivadores de la ciencia de nuestra lista en la Escuela Veterinaria de Madrid (7), el Jardín Botánico (4), los Colegios de Farmacia (4), la Escuela de Minas de Almadén (4), el Real Estudio de Mineralogía (3); así como en las diferentes Escuelas de diversas ciencias creadas por la Junta de Comercio de Barcelona (5), entre otros centros docentes no universitarios de enseñanza superior. Dentro del apartado "otros establecimientos educativos», hemos agrupado diversos establecimientos de enseñanza elemental, tales como el Colegio de Sordomudos de Madrid, la Casa de Caballeros Pajes, el Seminario de Nobles de Madrid, el Instituto Asturiano, colegios de prime- 
ras letras y liceos. Entre todos ellos, destacan los Reales Estudios de San Isidro de Madrid, donde trabajaron seis de los cultivadores de la ciencia estudiados.

Los miembros del Ejército constituyen otro grupo importante, dentro de los cultivadores de la ciencia españoles de nuestro período, tal vez como manifestación de la "militarización de la ciencia» española del siglo XVIII, de la que han hablado diversos autores (25): Además de los 56 individuos de este grupo, también debemos tener en cuenta los numerosos médicos, cirujanos y boticarios militares así como los profesores de Colegios de Cirugía dependientes del ejército que hemos contabilizado en los apartados anteriores. Centrando nuestra atención en el grupo de militares no médicos, hemos de destacar el importante peso de los militares de la Armada que constituyen más del $50 \%$ del total.

Los cultivadores de la ciencia que pertenecían al clero constituyen un grupo muy reducido dentro del conjunto total, lo cual contrasta con la posición privilegiada que la Iglesia tuvo en otros momentos en la actividad científica. El resto de los grupos tienen una importancia mucho menor, y tan sólo merece destacarse el grupo de juristas, empleados en diversas audiencias, cancillerías, etc. El último grupo incluye un elevado número de profesiones y ocupaciones a las que corresponden menos de dos cultivadores de la ciencia de nuestra lista. Entre ellas destacan los empleados en diversas instituciones científicas (13) como colectores de minerales, bibliotecarios, delineantes, grabadores, etc. así como los empleados en diversos puestos de la administración pública (7).

Resulta muy interesante estudiar las principales áreas científicas cultivadas por cada uno de estos grupos de profesiones y ocupaciones que hemos visto anteriormente. Hemos tratado de resumir esta información en la tabla VI. Como puede comprobarse, existen áreas científicas como la medicina, cirugía y farmacia, que eran cultivadas mayoritariamente por individuos cuya dedicación profesional estaba muy relacionada con el área científica cultivada. Así, del total de 179 cultivadores de esta área cuya profesión u ocupación conocemos, la mayor parte (124) de ellos estaban dedicados a una profesión u ocupación relacionada con la sanidad, tanto civil como militar, y el otro grupo importante (53) se dedicaba a la docencia de esta materias, bien en los Colegios de Cirugía y Farmacia o en las Universidades. Algo semejante ocurre con las áreas de ingeniería militar, cultivada, como es lógico, por militares, y la veterinaria, que fue cultivada por albéitares y profesores de la Escuela de Veterinaria en su mayoría. Del mismo modo, prácticamente la totalidad de los cultivadores de la 
TABLA VI

Areas cientificas cultivadas y ocupaciones y profesiones de los cultivadores de la ciencia

\begin{tabular}{lrrrrrrrr}
\hline \multicolumn{1}{l}{ Materias/Ocupaciones } & $\begin{array}{l}\text { Ocup. } \\
\text { sanit. }\end{array}$ & Profesor & Militar & $\begin{array}{c}\text { Ecle } \\
\text { siástico }\end{array}$ & Jurista & $\begin{array}{l}\text { Ing. } \\
\text { civil }\end{array}$ & Albéitar & Otros \\
\hline Medicina & 124 & 53 & - & 6 & - & - & - & 2 \\
Química & 15 & 23 & 5 & 3 & 2 & 2 & - & 15 \\
Historia natural & 9 & 17 & 2 & 7 & 1 & - & - & 9 \\
Matemáticas & - & 17 & 13 & 4 & - & - & - & 2 \\
Geografía & 1 & 5 & 12 & 4 & 4 & 1 & - & 3 \\
Astronomía & - & 5 & 7 & 3 & 2 & - & - & 4 \\
Agronomía & - & 6 & 2 & 2 & 5 & - & - & 7 \\
Náutica & - & 1 & 20 & - & - & - & - & - \\
Ingeniería civil & - & 9 & - & 2 & - & 2 & - & 4 \\
Veterinaria & - & 8 & 3 & - & - & - & 3 & - \\
Física & - & 5 & 1 & 5 & - & 1 & - & 1 \\
Ingeniería militar & - & - & 11 & - & - & - & - & - \\
Historia Ciencia & 1 & 3 & 1 & 2 & - & - & - & - \\
Ciencia en general & - & 1 & - & 1 & 2 & - & - & - \\
\hline
\end{tabular}

Fuente: Bertomeu SÁNCHEz (1993).

náutica son militares, en su mayoría de la Armada. También en la producción de obras matemáticas tienen un papel importante los militares que, junto con los maestros de los primeros niveles de la enseñanza, reúnen la mayor parte de estos cultivadores de la ciencia (27).

Otras áreas científicas como la química y sus aplicaciones eran cultivadas por individuos con muy diversas profesiones y ocupaciones, destacando entre éstas las de profesor de diversas instituciones relacionadas con la minería o mineralogía (Estudio de Mineralogía, Colegio de Minas de México, Escuela de Minas de Almadén), los Colegios de Cirugía y Farmacia, así como diversos individuos que hemos incluido en el grupo de profesiones y ocupaciones sanitarias, especialmente boticarios. También encontramos cultivadores de la química entre diversos empleados públicos (dirección de minas, director de la Casa de la Moneda, director fábri- 
cas salitre) y miembros de instituciones científicas no dedicados a la docencia (colectores, ayudantes de laboratorio).

Por su parte, la historia natural fue cultivada por un número importante de boticarios y clérigos, así como por los profesores del Jardín Botánico de Madrid. La agronomía fue cultivada también por autores con muy diversas ocupaciones y profesiones, desde profesores y empleados de Jardines Botánicos hasta juristas, militares y clérigos. Los cultivadores de la geografía y la astronomía, aunque mayoritariamente militares, incluyen también a profesores de diversos niveles, juristas y clérigos.

En definitiva, el análisis de estos datos confirma nuestra hipótesis inicial que hemos adelantado en el apartado "material y métodos». La actividad científica en la sociedad española de principios del siglo XIX fue desarrollada por un grupo muy diverso de individuos dedicados a distintas profesiones y ocupaciones, las cuales tenían más o menos relación con el área científica cultivada. Por lo tanto, resulta conveniente tener en cuenta que, al estudiar a los cultivadores de la ciencia de estos años, no estamos realizando un estudio de una comunidad profesional, con instituciones dedicadas a su formación en un área del saber determinada y con un conjunto de roles definidos dentro de la sociedad. En realidad, al hablar de "cultivadores de la ciencia» nos estamos refiriendo a un grupo muy heterogéneo de individuos cuya característica común fue la publicación de, al menos, una obra científica durante el período estudiado. Por ello, al estudiar sus actitudes políticas en el siguiente apartado, es necesario considerar la gran diversidad de situaciones en las que se encontraron los cultivadores de la ciencia durante la Guerra de la Independencia, lo cual hace sumamente difícil su estudio comparado.

\subsection{Actitudes politicas de los cultivadores de la ciencia frente al} gobierno de José I

Como hemos afirmado, el estudio de las actitudes políticas de los cultivadores de la ciencia durante el primer tercio del siglo XIX resulta de gran interés para comprender la situación de estos individuos durante el reinado de Fernando VII (1814-1833), caracterizado, entre otras cosas, por la persecución a liberales y afrancesados. Nos ocuparemos aquí de analizar la actitud de los cultivadores de la ciencia frente al gobierno de José I, con el objetivo de estudiar las características del grupo que ha sido denominado como «científicos afrancesados». Antes de exponer los resultados, de- 
bemos precisar el significado que otorgamos en este trabajo al término "afrancesado".

Como es sabido, el término "afrancesado" fue utilizado durante los años de la Guerra de la Independencia para hacer referencia a un grupo muy amplio de individuos, desde aquéllos que colaboraron con el gobierno de José I, hasta los partidarios de las Cortes de Cádiz, acusados de estar influidos por las ideas revolucionarias de Francia (26). Ya en 1817, Félix Reinoso, que se encontraba exilado en Francia, criticó el uso de este término polisémico por parte de determinados grupos políticos para criticar al grupo de ideas contrarias. Es interesante reproducir sus palabras:

"Todos los partidos, que se han suscitado en nuestra revolución, han procurado desacreditarse mutuamente por afrancesados. Los insurgentes de América han tratado al gobierno y á los patriotas europeos, como agentes de los Franceses... Los Europeos de su parte dicen que las turbulencias de América se han agitado por los Franceses... Los liberales, gloriándose de su patriotismo incorruptible... colocan en sus periódicos los nombres de afrancesados, juramentados y serviles baxo el mismo predicamento... [Por su parte, el partido servil] ha mirado siempre á los liberales como una facción francesa; porque adoptan los principios de su revolución, porque predican las ideas de sus escritores, porque han promovido muchas determinaciones semejantes a los decretos del rey intruso, porque desfavorecen los establecimientos de piedad. Los periódicos serviles están llenos de recriminaciones, y hay uno, que es el Filósofo de antaño, dedicado solamente á manifestar que los liberales son francmasones y afrancesados. ¿Qué sima es esta, donde todos los Españoles han caído? Qualesquiera que sean sus opiniones, qualquiera que el clima de su morada; americanos y europeos, leales é indiferentes, liberales y sèrviles, todos son, todos se apellidan afrancesados» (27).

Posteriormente, los estudios históricos sobre esta época han utilizado el término "afrancesado" con diversos sentidos, desde la simpatía por las ideas francesas hasta la colaboración con el gobierno de José I, lo cual ha generado cierta confusión que ha sido criticada por diversos estudiosos del tema. En concreto, la confusión entre afrancesamiento político (colaboración con el gobierno de José I) y afrancesamiento cultural ha resultado especialmente desorientadora para los estudios sobre la actitud política de los autores científicos. En muchas ocasiones, se ha tratado de explicar la colaboración de numerosos autores científicos con el gobierno 
de José I por el poderoso influjo que ejerció el pensamiento científico francés del momento sobre muchos de ellos. Al margen de la validez de este tipo de afirmaciones, es necesario señalar que el contacto cultural con Francia y la colaboración con el gobierno de José I son dos fenómenos diferentes, que no se pueden poner en relación sin realizar las oportunas investigaciones (28).

Nosotros utilizaremos el término "afrancesado" como sinónimo de colaboracionista o partidario del gobierno de José I, entendida esta colaboración en sentido amplio, desde los colaboradores directos del gobierno de José I, pasando por los de la administración central, hasta los diversos funcionarios de los pueblos y ciudades que tuvieron que prestar el juramento de fidelidad al nuevo rey (29). Por lo tanto, incluimos dentro de este grupo toda una serie de actitudes políticas frente al gobierno de José I, desde los que colaboraron libremente con el gobierno afrancesado, plenamente convencidos de que se trataba de la mejor opción, hasta aquéllos que, por diversos motivos, fueron obligados a prestar juramento al gobierno afrancesado y colaborar con él. En el bando opuesto, colocamos a todos aquéllos que se opusieron al gobierno de José I, desde los que participaron activamente en la lucha contra los franceses hasta los que residieron o se trasladaron a las zonas no controladas por el gobierno afrancesado, incluyendo tanto a los partidarios del gobierno absolutista de Fernando VII como a los de la Constitución de 1812.

La distinción entre estos dos grupos, partidarios y detractores del gobierno de José I, no resulta sencilla al aplicarla a los cultivadores de la ciencia. Nuestro primer problema ha sido el escaso conocimiento de las biografías de muchos de estos individuos, especialmente de aquéllos cuya obra científica ha sido considerada menos importante. En segundo lugar, muchos de estos datos biográficos proceden de trabajos con un marcado carácter hagiográfico, como elogios académicos o discursos conmemorativos que, en algunos casos, han intentado evitar informar sobre la colaboración del individuo ensalzado con el gobierno afrancesado, por considerar esta colaboración como un acto inmoral o antipatriótico. Por ello, ha resultado muy valioso para esta investigación el uso de la documentación del Ministerio del Interior afrancesado, donde aparecen muchas de las referencias a estos cultivadores de la ciencia, entre ellas los juramentos de fidelidad de los miembros de diversas instituciones científicas.

Con todo este material hemos conseguido obtener información sobre la actitud política de 284 cultivadores de la ciencia quedando un grupo de 199 que no hemos podido clasificar. Los resultados aparecen en la tabla

Asclepio-Vol. XLVI-1-1994 
VII. Además de los grupos donde incluimos por separado a partidarios y contrarios del gobierno de José I, hemos establecidos dos grupos más, «no clasificables» y "dudosos». Dentro del grupo de «no clasificables» hemos incluido a todos aquellos autores científicos que no se encontraban en la península durante la guerra y que no tuvieron que pronunciarse sobre su colaboración con el gobierno afrancesado. La mayor parte de ellos - 36corresponde a autores que residían en las colonias españolas y que, por lo tanto, se encontraban en una situación política bastante diferente, relacionada con los movimientos de independencia de estos territorios. En el grupo de dudosos hemos incluido once autores cuyos datos biográficos son contradictorios y no permiten su clasificación en uno de los anteriores grupos.

Los datos de la tabla VII muestran que el número de cultivadores de la ciencia que se opusieron al gobierno afrancesado fue mucho mayor (aproximadamente el doble) que el de sus partidarios. Resulta interesante estudiar estas actitudes políticas de acuerdo con la edad de los cultivadores de la ciencia durante este período, tal y como aparecen reflejadas en la tabla VIII.

Los datos que hemos podido reunir indican que la relación entre el número de autores científicos partidarios y el de contrarios al gobierno

TABLA VII

Actitud política de los cultivadores de la ciencia

\begin{tabular}{|c|c|c|}
\hline $\begin{array}{l}\text { Actitud política frente } \\
\text { al gobierno de José I }\end{array}$ & Número & Porcentaje \\
\hline Contrarios al gobierno de José I & 147 & 51,76 \\
\hline Afrancesados & 79 & 27,81 \\
\hline No clasificables & 47 & 16,55 \\
\hline Dudosos & 11 & 3,87 \\
\hline TOTAL & 284 & 100,00 \\
\hline
\end{tabular}

Fuente: BERTOMEU SÁNCHEZ (1993).

Asclepio-Vol. XLVI-1-1994 
TABLA VIII

Actitud política y edad de los cultivadores de la ciencia

\begin{tabular}{|c|c|c|c|c|}
\hline Fecha de nacimiento & $\begin{array}{l}\text { Antes de } \\
1770\end{array}$ & $1770-1785$ & $\begin{array}{c}\text { Después de } \\
1785\end{array}$ & Sin datos \\
\hline $\begin{array}{l}\text { Actitud política frente } \\
\text { al gobierno de José I: }\end{array}$ & & & & \\
\hline Contrarios al gobierno & 50 & 65 & 26 & 6 \\
\hline Afrancesados & 33 & 36 & 6 & 4 \\
\hline No clasificables & 17 & 6 & 1 & 23 \\
\hline Dudosos & 7 & 4 & - & - \\
\hline Sin datos & 40 & 34 & 31 & 94 \\
\hline TOTAL & 147 & 145 & 64 & 127 \\
\hline
\end{tabular}

Fuente: BERTOMEU SÁNCHEz (1993).

de José I varió de acuerdo con las distintas edades de estos individuos. En los dos primeros grupos, todos los mayores de 25 años, el número de opositores al gobierno afrancesado es algo menos del doble que el número de los partidarios, mientras que en el grupo de los cultivadores de la ciencia más jóvenes, el porcentaje de opositores es mucho mayor sumando algo más de cuatro veces el número de cultivadores de la ciencia afrancesados en este grupo de edad. Las causas de esta relación pueden explicarse teniendo en cuenta las características generales de estos grupos de edad y las características del grupo que hemos denominado "afrancesados». En primer lugar, es necesario considerar que numerosos jóvenes universitarios formaron batallones patrióticos para combatir a los franceses o se inscribieron en el ejército trabajando como ayudantes de farmacia, médicos, cirujanos, ingenieros, etc. Este reclutamiento sólo se produjo en el bando que luchaba contra las tropas francesas, puesto que el gobierno de José I no llegó a disponer de un ejército nacional importante (30). Por otra parte, los autores científicos más jóvenes no habían accedido, en la mayor parte de los casos, a cargos institucionales que dependían del gobierno. Por ello, al contrario que los otros grupos de 
edad, los más jóvenes no fueron obligados a prestar juramento de fidelidad para permanecer en sus cargos.

Otra característica que diferencia a los cultivadores de la ciencia partidarios y contrarios del gobierno de José I es el lugar donde residieron durante la guerra. Más de tres cuartas partes de los cultivadores de la ciencia "afrancesados» residieron durante la guerra en Madrid (77\%). El resto de este grupo se reparten entre trece ciudades y pueblos más, de los que únicamente destaca Valencia con tres residencias. Los opositores del gobierno afrancesado estaban más dispersos por la geografía peninsular (más de cincuenta ciudades y pueblos diferentes), puesto que muchos de ellos se vieron obligados a desplazarse de un lugar a otro, huyendo de las tropas francesas. Como es lógico, la ciudad con mayor número de residencias de este grupo es Cádiz que recoge algo más de la quinta parte de las mismas $(21 \%)$. Las restantes cuatro quintas partes se reparten entre más de cincuenta ciudades y pueblos entre las que destacan Madrid (7\%), Valencia y Sevilla (6\%). Podemos afirmar, por lo tanto, que los cultivadores de la ciencia que hemos denominado «afrancesados» se agruparon mayoritariamente alrededor de una sola ciudad, Madrid, mientras que los opositores al régimen de José I se dispersaron por un elevado número de ciudades, de entre las que destaca Cádiz.

También podemos encontrar diferencias entre las áreas científicas cultivadas por los partidarios del gobierno afrancesado y los opositores al mismo, tal y como se puede comprobar en la tabla IX.

En ambos casos, las materias científicas mayoritariamente cultivadas son la medicina, cirugía y farmacia y la química, aunque en el bando afrancesado el porcentaje de cultivadores de la medicina es bastante más bajo que la media total. En otras materias, la diferencia entre ambos grupos es mucho mayor. Así, entre los afrancesados apenas encontramos cultivadores de la náutica o de la ingeniería militar, siendo mucho menor el porcentaje de cultivadores de la astronomía que la media. Sin embargo, materias como la historia natural y la agronomía y zootecnia tienen un porcentaje de cultivadores mayor que la media. Como es fácil suponer, en el bando contrario a José I, la situación es, aproximadamente, la imagen especular de la del bando afrancesado. El porcentaje de cultivadores de la ingeniería militar, las matemáticas y la asstronomía es mayor que el de la media total mientras que es ligeramente inferior el porcentaje de cultivadores de la historia natural y botánica y la agronomía y zootecnia. Las causas para esta distribución deben buscarse en la diversas ocupaciones y 
TABLA IX

Areas cultivadas y actitud política

\begin{tabular}{|c|c|c|c|c|}
\hline \multirow[b]{2}{*}{ Materias científicas } & \multicolumn{2}{|c|}{ Afrancesados } & \multicolumn{2}{|c|}{ Contrarios al gobierno } \\
\hline & $N .^{\circ}$ & $\%$ & $N .^{o}$ & $\%$ \\
\hline Medicina & 30 & 28,85 & 70 & 35,53 \\
\hline Química & 15 & 14,42 & 24 & 12,18 \\
\hline Historia Natural & 12 & 11,54 & 14 & 7,11 \\
\hline Geografía & 9 & 8,65 & 16 & 8,12 \\
\hline Agronomía y zootecnia & 7 & 6,73 & 5 & 2,54 \\
\hline Matemáticas & 7 & 6,73 & 15 & 7,61 \\
\hline Ingeniería civil & 7 & 6,73 & 8 & 4,06 \\
\hline Veterinaria & 5 & 4,81 & 5 & 2,54 \\
\hline Astronomía & 3 & 2,88 & 10 & 5,08 \\
\hline Náutica & 3 & 2,88 & 13 & 6,60 \\
\hline Física & 2 & 1,92 & 5 & 2,54 \\
\hline Historia de la ciencia & 2 & 1,92 & 2 & 1,02 \\
\hline Ingeniería militar & . 1 & 0,96 & 8 & 4,06 \\
\hline Ciencia en general & 1 & 0,96 & 2 & 1,02 \\
\hline TOTAL & 104 & 100,00 & 197 & 100,00 \\
\hline
\end{tabular}

Fuente: BERTOMEU SÁNCHEz (1993).

profesiones que tenían los cultivadores de cada una de estas materias, tal y como queda reflejado en la tabla X.

Como se puede deducir de los datos de esta tabla, la mayor parte de los cultivadores de la ciencia que pertenecían al ejército, incluyendo los médicos, cirujanos y boticarios militares, se posicionaron contra el gobierno afrancesado. Muchos de ellos se inscribieron en el ejército por estas fechas y otros pertenecían a él antes de la guerra por lo que, al igual que la mayor parte del ejército español, participaron en la lucha contra la ocupación francesa (31). Ello explica la escasa presencia en el bando afrancesado de cultivadores de áreas como la ingeniería militar o la náutica que eran mayoritariamente cultivadas por miembros del ejército. La mayor parte de las ocupaciones de los cultivadores de la ciencia partidarios de 
TABLA X

Actitud política y profesiones y ocupaciones

\begin{tabular}{lcc}
\hline Profesiones y ocupaciones & $\begin{array}{c}\text { Partidarios del } \\
\text { gobierno José I }\end{array}$ & $\begin{array}{c}\text { Contrarios al } \\
\text { gobierno de José I }\end{array}$ \\
\hline Profesores & 3 & 5 \\
$\quad$ Profesor universidad & 22 & 31 \\
$\quad$ Profesor instituciones científicas & 6 & 7 \\
$\quad$ Otros profesores & 9 & 21 \\
Profesiones y ocupaciones sanitarias & - & 5 \\
$\quad$ Médico & - & 13 \\
$\quad$ Cirujano & 5 & 6 \\
$\quad$ Boticario & 6 & 36 \\
$\quad$ Cargos administración sanitaria & 7 & 2 \\
Militares & 3 & 5 \\
Eclesiásticos & 3 & 1 \\
Juristas & 1 & 9 \\
Ingenieros civiles & 13 & 141 \\
Albéitares & & \\
Otros & 78 & \\
\hline TOTAL & & \\
\hline
\end{tabular}

Fuente: BERTOMEU SÁNCHĖZ (1993).

José I corresponden a cargos dentro de instituciones científicas, en especial de Madrid. También destaca un número importante de clérigos y diferentes cargos de la administración de José I (prefectos, intendentes, jefes de sección), que explica el abundante número de cultivadores de la historia natural y la agronomía y zootecnia (32). En definitiva, podemos caracterizar, a grandes rasgos, el conjunto de cultivadores de la ciencia partidarios de José I como autores científicos consagrados, de edad superior a 25 años, generalmente con alguna obra publicada antes de la guerra y miembros de alguna institución científica, principalmente de las establecidas en Madrid. Sin embargo, a pesar de estas características generales, las diferencias entre los miembros de este grupo son muy importantes, variando desde los que colaboraron en un primer momento, ocupando cargos im- 
portantes dentro del estado bonapartista, (José María Lanz, Francisco Antonio Zea, Domingo Badía Leblich, José Garriga y Buach, Antonio Cibat, Tomás García Suelto, Francisco Angulo, José de Mazarredo, etc.) hasta los que se limitaron a permanecer en sus puestos, por lo que, forzada o voluntariamente, prestaron el juramento de fidelidad al nuevo rey (Pedro Gutiérrez Bueno, Claudio y Esteban Boutelou, José Mariano Mociño, Antonio de Gimbernat, etc.). En cualquier caso, nuestros datos indican que las razones que llevaron a este grupo de individuos a optar por un determinada actuación política durante estos años están más relacionadas con su posición social, su profesión y ocupación, que con sus estudios y trabajos científicos. Tal y como han puesto de manifiesto estudiosos de otros campos históricos, tampoco en historia de la ciencia resulta posible asimilar el «afrancesamiento cultural» con el «afrancesamiento político».

\section{NOTAS}

(1) El resultado de este trabajo ha sido la tesis doctoral Bertomeu SÁnChEz (1993) que ha sido posible, en parte, gracias a una beca del Ministerio de Educación y Ciencia, dentro del programa de formación de personal investigador.

(2) Sobre la aplicación de la prosopografía en la historia de la ciencia véase el trabajo de SHAPIN Y THACKRAY (1974).

(3) Una revisión sobre el conjunto de estos estudios en Lö́PEZ Piñero (1987) y LóPEZ Piñero; Terrada (1993). Un ejemplo de los excelentes resultados de la aplicación de la prosopografía para el estudio de la ciencia española es el capítulo II de LóPEz PIÑERo (1979), 47-88.

(4) Seguimos aquí el clásico trabajo de BEN-DAvid (1976) así como diversos estudios dedicados a la situación en Francia durante este período como los de GILLESPIE (1980), Crosland (1975), Hanh (1975), Outram (1980) y, más recientemente, Dhombres, N.; DHOMBRES, J. (1989).

(5) De acuerdo con ello, las materias consideradas han sido: Agronomía y zootecnia, astronomía, ciencias extraacadémicas, física, historia natural, geografía, ingeniería civil y militar, matemáticas, medicina y farmacia, náutica, veterinaria y química. Una discusión más detallada de este punto en BERTOMEu SÁNCHEZ (1993), 19 y ss.

(6) PRICE (1978), especialmente "A definition of research", 79-83.

(7). Sobre este archivo, v. López Terrada; Pla Vall (1982) y la reseña biográfica de GÓMEZ MARTf (1923) que contiene información sobre la producción impresa y manuscrita de Rodrigo Pertegás.

(8) Esta documentación ha sido estudiada en la tesis doctoral de MIco NAVARRO (1986). 
(9) No podemos resumir aquí esta abundante bibliografía que hemos recogido en Bertomeu Ś́nchez (1993), 363-380. Citaremos, a modo de ejemplo, el estudio de BarreiRo (1944, reed. 1992) sobre el Real Gabinete de Historia Natural, TiNoco (1951) sobre el Observatorio Astronómico, Puerto SARMIENTo (1988) sobre el Jardín Botánico, Folch Jou (1977) sobre el Real Colegio de Farmacia así como los múltiples trabajos dedicados a los Colegios de Cirugía como el de Aparicio Simón (1956) o UsandizaGa (1964).

(10) Sobre la estructura del Estado proyectado por el gobierno de José I, es obligatorio consultar la obra de Mercader RiBa (1971) (1983). Sobre el Ministerio del Interior, v. MERCADER RIBA (1982).

(11) La recopilación legislativa más importante es el PRONTUARIo (1810-1812), completado con la de Ríos, J.M. (1854).

(12) La descripción completa de esta documentación se encuentra en BERTOMEU SÁNCHEZ (1993), 5-11.

(13) Especialmente en las secciones «Papeles reservados de Fernando VII» y la «Sección General», serie "Gobierno Intruso».

(14) En la sección Consejos, legajos 17783-17888 y 49613-49616. En la sección «Estado" se encuentran varios expedientes personales formados en juicios contra los afrancesados, legajos 3091-3095, 3101, 3108, 3116, etc.

(15) En la sección "Gracia y Justicia», serie "Gobierno Intruso».

(16) Legajo D-1551

(17) Dentro de la sección «Archives Joseph Bonaparte».

(18). Estos libros de registros se encuentran en el Archivo del Palacio Real. Gobierno Intruso. Libros 2208 y 2209. y Archivo General de Simancas. Gracia y Justicia. Gobierno Intruso. Legajo 1090. Más datos sobre la documentación conocida del gobierno afrancesado en las obras de Mercader Riba (1983), Artola (1976), Tourtier-Bonazzi (1982) y AY. MES (1989).

(19) DanVila y Collado (1880), t. VI, 688-690. Esta lista corresponde a una estructura de un Instituto de Ciencias y Letras, semejante al existente en Francia durante esos años. Aunque diversas razones muestran su autenticidad, no todos los individuos que aparecen en esta lista pueden ser considerados como afrancesados. Sobre este tema v. BERTOMEU SÁNCHEZ (1993), 65-69.

(20) Seguimos en este punto el análisis generacional de López Piñero, según aparece en varios de sus trabajos.. Véase, por ejemplo, LÓPEZ PIÑERO, J.M. (1964), 64; LóPEZ PIÑERO (1979b), 75-76 y, más recientemente, el volumen colectivo dirigido por LÓPEZ PIÑERO (1992).

(21) Sobre la situación de Cádiz, Valencia y Barcelona durante la guerra, v. Solfs (1969), CRUZ Román (1968) y MERCADER Riba (1949).

(22) Bertomeu SÁNCHEZ (1993), 44-179.

(23) BERTOMEU SÁNCHEZ (1993), 216.

(24) Bertomeu SÁNCHEZ (1993), 216-217.

(25) Véase, por ejemplo, Selles; Lafuente; Peset (1988).

(26) Véase, por ejemplo, JuRETSCHKE (1986), 51-59; Artola (1976), 50-56.

(27) EXAmen (1818), 264-266.

(28) Véase, por ejemplo, JuRetschKe (1986), 59; ARTola (1976), 23; Una crítica a la confusión entre afrancesado político y cultural en Dupuis (1963), 143 y ss. También resul- 
ta interesante para este tema la biografía de Meléndez Valdés realizada por DEMERson (1971), t. II, 329-344.

(29) Seguimos aquí el uso de este término que hace MERCADER Riba en sus obras (1971) (1983). Véase MERCADER RIBA (1971), 7.

(30) MERCADER RiBa (1983), 294-312.

(31) Mercader Riba (1983), 294-301. José Bonaparte nunca llegó a tener un auténtico ejército formado por tropas españolas.

(32) Existen pocos estudios generales sobre la composición social de los afrancesados. Algunos datos se pueden obtener de los trabajos de Dufour (1973) y BRINEs (1984). Para un estado de la cuestión, véase Aymes (1987), 118.

\section{BIBLIOGRAFIA}

Álvarez Sierra, J. (1963), Diccionario de autoridades médicas, Madrid, Editora Nacional. ANTÓN RAMfREZ, B. (1865), Diccionario de Bibliografía agronómica, y de toda clase de escrito relacionados con la Agricultura, Madrid, M. Rivadeneyra.

Aparicio Simón, J. (1956), Historia del Real Colegio de San Carlos de Madrid, Madrid, Aguilar.

ARtola, M. (1976), Los afrancesados, Madrid, Turner.

ARtola, M.(dir) (1991), Enciclopedia de Historia de España dirigida por... IV. Diccionario biográfico, Madrid, Alianza Editorial.

AYMES, J.R. (1989), L'Espagne en mouvement (1766-1814). Essai bibiliographique. En: AYMES, J.R ; GIL Novales, A. ; Oliveira Ramos, L.A. (eds.) Les revolutions dans le monde iberique, 1766-1834, Talence, Presses Universitaires de Bordeaux, pp. 13-140.

Barreiro, A.J. (1992), El Museo Nacional de Ciencias Naturales, Madrid, Museo de Ciencias Naturales.

Ben-David, J. (1974), El papel de los científicos en la sociedad. Un estudio comparativo. México, Editorial Trillas.

BERTOMEU SÁNCHEZ, J.R. (1993) La actividad científica en España bajo el reinado de José 1 (1808-1813). Un estudio de las instituciones, autores y publicaciones científicas a través de la documentación del gobierno afrancesado. Valencia. Tesis Doctoral.

BONET CORREA, A.(dir) (1980), Bibliografía de arquitectura, ingeniería y urbanismo en España (1498-1880), Madrid, Turner.

Bover, J.M. (1868), Biblioteca de escritores baleares, Palma, Imprenta de P.J. Gelabert.

BRINES, J. (1984), Aproximación al estudio sociológico de los afrancesados en el País valenciano. En: AA.VV. Les Espagnols et Napoleon: actes du Colloque international d Aixen-Provence, Aix-en-Provence, Univ. Provence. Etudes Hispaniques n ${ }^{\circ}$ 7, pp. 269-275.

Calbet i Camarasa, J.M.; Corbella i Corbella, J. (1981-83), Diccionari biogrăfic de metges catalans, Barcelona, II Congrés Hist. Medicina Catalana, 3 vols.

CAPEL, H.; et al. (1983), Los ingenieros militares, siglo XVIII. Repertorio biográfico e inventario de su labor científica y espacial, Barcelona, Universidad. Geocrítica. Cuadernos apoyo. 
Colmeiro, M. (1858), La Botánica y los botánicos de la Península Hispano-lusitana. Estudios bibliográficos y biográficos, Madrid, M. Rivadeneyra.

CORMinAS, J. (1849), Suplemento al diccionario crítico de los escritores catalanes, Burgos, Imprenta de Arnaiz.

Crosland, M. (1975), Development of a professional career in science in France. En: CrosLAND, M. (ed.) The emergence of Science in Western Europe, London, pp. 139-161.

CRUz RomÁn, N. (1968), Valencia Napoleónica, Valencia.

Chinchilla, A. (1841-46), Anales históricos de la medicina en general y biográfico-bibliográficos de la española en particular, Valencia, Imprenta de López y Cia. 4 vols.

Danvila y Collado, M. (1880), Relación del profesorado de España elegido por José Napoleón Bonaparte, con el juicio de cada uno de sus profesores. En: Del poder civil en España, t. VI, pp. 688-90.

Demerson, G. (1971), Don Juan Melendez Valdés y su tiempo, Madrid, Taurus, 2 vols.

Dhombres, J.; Dhombres, N. (1989), Naissance d'un nouveau pouvoir. Sciences et savantes en France (1793-1824), Paris, Payot.

Dufour, G. (1973), Infidencia et afrancesamiento: quelques donnés statistiques, En: Etudes d' Histoire litteraire et lingüistique. Rouen. Centre Ibero-americain de la Université.

DupuIS, L. (1963), A propos d' afrancesamiento, Caravelle, 1, 141-157.

Elías de Molins, A. (1972), Diccionario biográfico y bibliográfico de escritores y artistas catalanes del siglo XIX, New York, 2 vols.

EXAMEN (1818)...de los delitos de infidelidad á la patria, imputados à los españoles sometidos baxo la dominación francesa. 2. ${ }^{a}$ edición, Burdeos, Juan Picard.

FernÁNDez de NAVARRete, M. (1851), Biblioteca marítima española, Madrid, Vda. de Galeno, 2 vols.

Folch Jou, G. (1977), El Real Colegio de Farmacia de San Fernando, Madrid.

GIL Novales, A. et al. (1991), Diccionario biográfico del trienio liberal, Madrid.

GILlisPIE, C.C. (1980), Science and Polity in France at the End of the Old Regime, Princenton.

Gómez Marti, P. (1923) El investigador histórico y médico valenciano José Rodrigo de Pertegás, Medicina Valenciana, 23, 1-23 y 33-51.

GÓMEZ URIEL, M.(ed.) (1884-86), Biblioteca antigua y nueva de escritores aragoneses de Latassa, Zaragoza, Imprenta de Calisto Ariño, 3 vols.

Hernández MoRejon, A. (1842-52), Historia bibliográfica de la medicina española, Madrid, Imprenta de la Viuda de Jordán e Hijos, 7 vols.

JURETSCHKE, H. (1986), Los afrancesados en la guerra de la Independencia, Madrid, Sarpe.

LÓPEZ PiÑERo, J.M. (1968), La literatura científica en la España Contemporánea. En: DiAz PlaJA, G. (dir) Historia General de las Literaturas Hispánicas, vol. VI, pp. 677-693.

López Piñero, J.M. (1979), Ciencia y técnica en la sociedad española de los siglos XVI y XVII, Barcelona, Labor.

LÓPEZ PIÑERo, J.M. (1979b), La marginación de la ciencia en la España Contemporánea. En: Gónzalez Bueno, P.; Jiménez Blanco, J.; López Piñero, J.M. Historia y sociología de la ciencia en España, Madrid, Alianza Editorial, pp. 72-93.

LÓPEZ PIÑERO, J.M. (1987), Los modelos de investigación historicomédica y las nuevas técnicas. En: LAfUente, A.; SAldañA, J.J. (dirs.) Nuevas tendencias. Historia de las ciencias, Madrid, CSIC, pp. 125-150.

Asclepio-Vol. XLVI-1-1994 
López Piñero, J.M.; García Ballester, L.;Faus Sevilla (1964), Medicina y sociedad en la España del siglo XIX, Madrid, Sociedad de Estudios y Publicaciones.

López Piñero, J.M.; Terrada, M.L. (1993), Veinte años de investigación bibliométrica en el Instituto de Estudios Documentales e Históricos sobre la Ciencia, Valencia, IEDHC.

LóPEZ PIÑERo, J. (ed.) (1992), La ciencia en la España del siglo XIX, Ayer, 7.

LÓPEZ Piñero, J.M. et al. (eds.) (1983), Diccionario histórico de la Ciencia Moderna en España, Barcelona, Península. 2 vols.

LóPeZ Terrada, M.L.; Pla Vall, E. (1982) Médicos valencianos del siglo XVIII en el archivo de José Rodrigo Pertegás. En: AA.VV. Estudios dedicados a Juan Peset Aleixandre. t. II, pp. 549-579.

MAFFeI, E.; RUA FigueroA, R. (1871-72), Apuntes para una Biblioteca española de libros, folletos y artículos, impresos y manuscritos, relativos al conocimiento de las riquezas minerales y a las ciencias auxiliares, Madrid.

MERCADER RIBA, J. (1949); Barcelona durante la ocupación francesa (1809-1814), Madrid, C.S.I.C.

Mercader Riba, J. (1971), José Bonaparte Rey de España: 1808-1813. Historia externa del reinado, Madrid, C.S.I.C.

Mercader Riba, J. (1982), La instauración del Ministerio del Interior bajo José Bonaparte, en 1809, Hispania, 150, 183-207.

MERCADER RIBA, J. (1983), José Bonaparte Rey de España (1808-1813). Estructura del Estado español Bonapartista, Madrid, C.S.I.C.

Mico, J. (1986) León Sánchez Quintanar (1801-1877). Vida, obra y biblioteca. Valencia, Universidad. Tesis doctoral (microficha).

Outram, D. (1980), Politics and vocation: French Science, 1793-1830, British Journal for the History of Science, 13 (43), 27-43.

Palau Claveras, A. (1973), Bibliografía hispánica de veterinaria y equitación, Madrid.

Pastor Fuster, J. (1827-30), Biblioteca Valenciana, Valencia, J. Ximeno i Mompie.

PricE, D.J.S. (1978) Toward a Model of Science Indicators. En: J. ElKana et al. Toward a Metric of Science, New York, Wiley, pp. 69-95.

Prontuario, (1810-12), ... de las leyes y decretos del rey nuestro señor don José Napoleón I, Madrid, 3 vols.

PUerto SARMIENTO, F.J. (1988), La ilusión quebrada: botánica, sanidad y politica científica en la España ilustrada, Barcelona-Madrid, C.S.I.C.

Ríos, J.M. (1854), Código español del reinado intruso de José Napoleón Bonaparte, o sea, colección de sus más importantes leyes, decretos e instituciones., Madrid.

RoldÁN GUERRERo, R. (1958-76), Diccionario biográfico y bibliográfico de autores farmacéuticos españoles, Madrid, Gráficas Valera. 4 vols.

Selles, M.; PeSeT, J.L.; LAfuente, A. (1987), Carlos III y la ciencia de la Ilustración, Madrid, Alianza Editorial.

Shapin, S.; THACKRaY, A. (1974), Prosopography as a Research Tool in History of Science, History of Science, 22, 1-28.

Solís, R. (1958), El Cádiz de las Cortes, Madrid, Instituto de Estudios Políticos.

SUÁREZ, C. (1939-59), Escritores y Artistas asturianos, Oviedo. 7 vols.

TEN Ros, A.E. (1990), Scientifiques et Francisés. Dependances intellectuelles des scientifiqus espagnols à la fin du XVIIIe siècle et au debut du XIXe. En: Actes du 114e Con- 
grés national des Sociétés Savantes. Section histoire des sciences et des technique, Paris, CTHS, 21-36.

Tinoco, J. (1951), Apuntes para la historia del Observatorio de Madrid, Madrid.

TORRES AMAT, P. (1836), Memorias para ayudar a formar un diccionario crítico de escritores catalanes..., Barcelona, Imprenta de J. Verdaguer.

TourtiER-BonaZZI, C. (1982), Archives de Joseph Bonaparte, roi de Naples, puis d'Espagne (381 AP), Paris, Archives Nationales.

Usandizaga Saraluce, M. (1964), Historia del Real Colegio de Cirugía de Barcelona, Barcelona. 